\title{
Gender-specific modulation of short-term neuroplasticity in the visual cortex induced by transcranial direct current stimulation
}

\author{
LEILA CHAIEB, ANDREA ANTAL, AND WALTER PAULUS \\ Department of Clinical Neurophysiology, Georg-August University, Göttingen, Germany
}

(Received August 30, 2007; AcCePted December 14, 2007)

\begin{abstract}
Transcranial direct current stimulation (tDCS) is a non-invasive method of modulating levels of cortical excitability. In this study, data gathered over a number of previously conducted experiments before and after tDCS, has been re-analyzed to investigate correlations between sex differences with respect to neuroplastic effects. Visual evoked potentials (VEPs), phosphene thresholds (PTs), and contrast sensitivity measurements (CSs) are used as indicators of the excitability of the primary visual cortex. The data revealed that cathodally induced excitability effects $10 \mathrm{~min}$ post stimulation with tDCS, showed no significant difference between genders. However, stimulation in the anodal direction revealed sex-specific effects: in women, anodal stimulation heightened cortical excitability significantly when compared to the age-matched male subject group. There was no significant difference between male and female subjects immediately after stimulation. These results indicate that sex differences exist within the visual cortex of humans, and may be subject to the influences of modulatory neurotransmitters or gonadal hormones which mirror short-term neuroplastic effects.
\end{abstract}

Keywords: Visual cortex, Neuroplasticity, tDCS, Gender-effects

\section{Introduction}

In the human brain, the visual and motor cortices continue to be popular models for the investigation of induced structural plasticity in the adult brain, as their effects can be measured by visual (VEPs and PTs) responses or motor evoked potentials (MEPs), respectively (Nitsche \& Paulus, 2000; Boroojerdi et al., 2002; Antal et al., 2004). There is growing evidence to suggest that neuroplastic changes are influenced by gender-specific pressures. Kuo et al. (2006) recently reported sex differences in the primary motor cortex after short-duration tDCS; female subjects showed prolonged after-effects of cathodal stimulation, while male subjects showed stronger anodally-induced after effects. Previous animal and human studies of neuroplasticity and sex differences centered on cognitive functions (Kimura, 2002; Juraska, 1998) where male and female populations are assessed for hormonal influences on cognitive functions (spatial navigation facial processing emotion and memory; (see Cahill, 2006 for review)), which are believed to underlie these differences in cognitive responses between the sexes.

Address correspondence and reprint requests to: Leila Chaieb, Department of Clinical Neurophysiology, Georg-August University, RobertKoch-Str. 40, 37075 Göttingen, Germany. E-mail: leilachaieb@med. uni-goettingen.de
Administration of polarizing currents to the rat cerebral cortex revealed the ability to induce prolonged changes in cortical excitability (Bindmann et al., 1964). In humans, changes in cortical excitability, induced by tDCS have been shown to precede more enduring plastic changes that are reversible and can be pharmacologically modulated (Lang et al., 2005; Nitsche et al., 2003b, 2006). Stimulation with tDCS applied to the motor cortex is polarity-dependent; anodal stimulation results in cortical excitation, while cathodal tDCS produces inhibition of activated cortical networks (Nitsche \& Paulus, 2000).

The visual cortex possesses the ability to undergo induced and spontaneous neuroplastic changes that have been demonstrated to lead to both short-term and, to potentiate, more permanent alterations at the synapse (Sherman \& Spear, 1982). tDCS over the primary visual cortex in an anodal direction reduces PTs, while stimulation in the cathodal direction reverses this effect (Antal et al., 2003). Also cathodal stimulation has an inhibitory effect on the N70 component of VEPs and anodal tDCS induces the reverse response. CS measurements also reflect cathodal effects (Antal et al., 2001). The duration of tDCS after-effects are able to outlast the stimulation period if administered for some minutes (Nitsche $\&$ Paulus, 2001) over the primary motor cortex; tDCS after-effects are not so enduring when applied to the primary visual cortex (Antal et al., 2004). Visual and motor cortices vary with regards to factors influencing neuroplasticity and the excitatory/inhibitory 
circuitries, gonadal sex hormones and neurotrophins which act upon them (Lund, 2002; Keller, 1993). Differences in cortical connections and neuronal membrane properties, including receptor expression, between the primary motor and visual cortices may also account for the contrasting responses to the application of tDCS.

In order to examine how neuroplasticity in the visual cortex is influenced, if at all by gender, data gathered from previous experiments involving tDCS modulation of PTs, VEPs, and CSs, were reviewed (Antal et al., 2001, 2003, 2004). Interactions between gender and tDCS stimulation were analyzed, in an attempt to reveal potential gender-bias in induced short-term neuroplastic effects.

\section{Materials and methods}

\section{Participants}

Data collected from previous experiments conducted with 46 healthy subjects, was re-analyzed to ascertain whether neuroplasticity is influenced by interactions with gender. Of the 46 subjects, 22 were male (average age 27.2 years, SD: \pm 5.4 ), and 24 were female (average age 23.6 years, SD: \pm 2.4 ). None of the participants were involved in more than one of the original studies. Participants were interviewed prior to the start of the experiment, about their state of health, and satisfied all the necessary conditions; no history of neurological or psychological disorders, metallic implants, drug abuse, or alcoholism. No subjects were taking regular medication at the time and during the experiment. All of the participants gave written and informed consent and all experiments were conducted in accordance with the Declaration of Helsinki, and with the approval of the ethics committee of the University of Goettingen, Germany.

tDCS was administered to all subjects for a 7 or $10 \mathrm{~min}$ period (Table 1), in both an anodal and cathodal direction, using a battery-driven constant current stimulator (Schneider Electronic, Gleichen, Germany). A pair of standard rubber electrodes $(5 \times$ $7 \mathrm{~cm}^{2}$ ) encased in saline-soaked synthetic sponges, were placed over the $\mathrm{Oz}$ (active electrode) and $\mathrm{Cz}$ (reference electrode) posi- tions. The applied current had an intensity of $1.0 \mathrm{~mA}$. Stimulation sessions were separated by one week to avoid interference between stimulations.

\section{Measurement of visual cortical excitability}

Three parameters were measured; PT, CS, and VEPs, as comparable indicators of visual cortical excitability (for technical details and protocols see Antal et al., 2001, 2003, 2004). Participants' PTs, VEPs, and CS were measured prior to, immediately after, and 10 min after cathodal and anodal tDCS stimulation. See Table 1 for a summary of measurements and subject characteristics.

\section{Statistics}

All data was standardized prior to analysis in order to gain a result relating to absolute change in cortical excitability (\% change) when compared to baseline activity prior to, immediately after, and $10 \mathrm{~min}$ post tDCS stimulation. Values over $100 \%$ are an increase in cortical excitability and represent an increase in visual sensitivity for CSs and an increase in amplitudes for VEPs, but a decrease in the threshold for the perception of phosphene (PTs).

Repeated measures ANOVA was performed on the calculated data dependent variable: \% change, within effects: STIMULATION (anodal, cathodal), TIME (0 and $10 \mathrm{~min}$ after), categorical factor: GENDER (male, female). Student's $t$-test (independent by GENDER) was used to explore significant differences between female and male subjects concerning anodal and cathodal post-stimulation values immediately after stimulation and $10 \mathrm{~min}$ post stimulation separately. Statistical significance was assumed at $p \leq 0.05$.

\section{Results}

Immediately after the anodal tDCS, both sexes appeared to be within the same range, however, at 10 min post-stimulation measurements, the female group still showed a strong facilitatory response, while the male group showed a marked inhibitory response. The means of male and female subjects have been tabulated, including stimulation direction, and duration (see Table 2).

Table 1. Stimulation paradigms and measurements ${ }^{\mathrm{a}}$

\begin{tabular}{|c|c|c|c|c|c|}
\hline Measurement & Sex & $\begin{array}{l}\text { Age } \\
\mathrm{SD} \pm\end{array}$ & $\begin{array}{l}\text { Number of } \\
\text { participants }\end{array}$ & $\begin{array}{c}\text { tDCS } \\
\text { stimulation polarity }\end{array}$ & $\begin{array}{l}\text { Duration of } \\
\text { stimulation }\end{array}$ \\
\hline \multirow[t]{2}{*}{ Visual Evoked Potential (VEP) } & M & $\begin{array}{r}26.5 \\
2.7\end{array}$ & 10 & Anodal and Cathodal & $10 \mathrm{~min}$ \\
\hline & F & $\begin{array}{r}24.0 \\
2.0\end{array}$ & 5 & Anodal and Cathodal & $10 \mathrm{~min}$ \\
\hline \multirow[t]{2}{*}{ Stationary phosphene (PT) } & M & $\begin{array}{r}29.6 \\
7.8\end{array}$ & 4 & Anodal and Cathodal & $10 \mathrm{~min}$ \\
\hline & $\mathrm{F}$ & $\begin{array}{r}23.2 \\
2.4\end{array}$ & 12 & Anodal and Cathodal & $10 \mathrm{~min}$ \\
\hline \multirow[t]{2}{*}{ Contrast measurement (CS) } & M & $\begin{array}{r}26.8 \\
6.9\end{array}$ & 8 & Anodal and Cathodal & $7 \mathrm{~min}$ \\
\hline & $\mathrm{F}$ & $\begin{array}{r}23.8 \\
2.8\end{array}$ & 7 & Anodal and Cathodal & $7 \mathrm{~min}$ \\
\hline
\end{tabular}

${ }^{\text {a }}$ Summary of stimulation paradigms and measurements obtained from participants, including sex and age characteristics. Cathodal and anodal stimulation was applied to all subjects, and varied only in duration. For the VEP (10 male, 5 female) and stationary phosphene measurements (4 male, 13 female), stimulation in both polarities lasted $10 \mathrm{~min}$, whilst for the contrast measurements $(8$ male, 9 female $)$ stimulation lasted only $7 \mathrm{~min}$, and again in both polarities. 


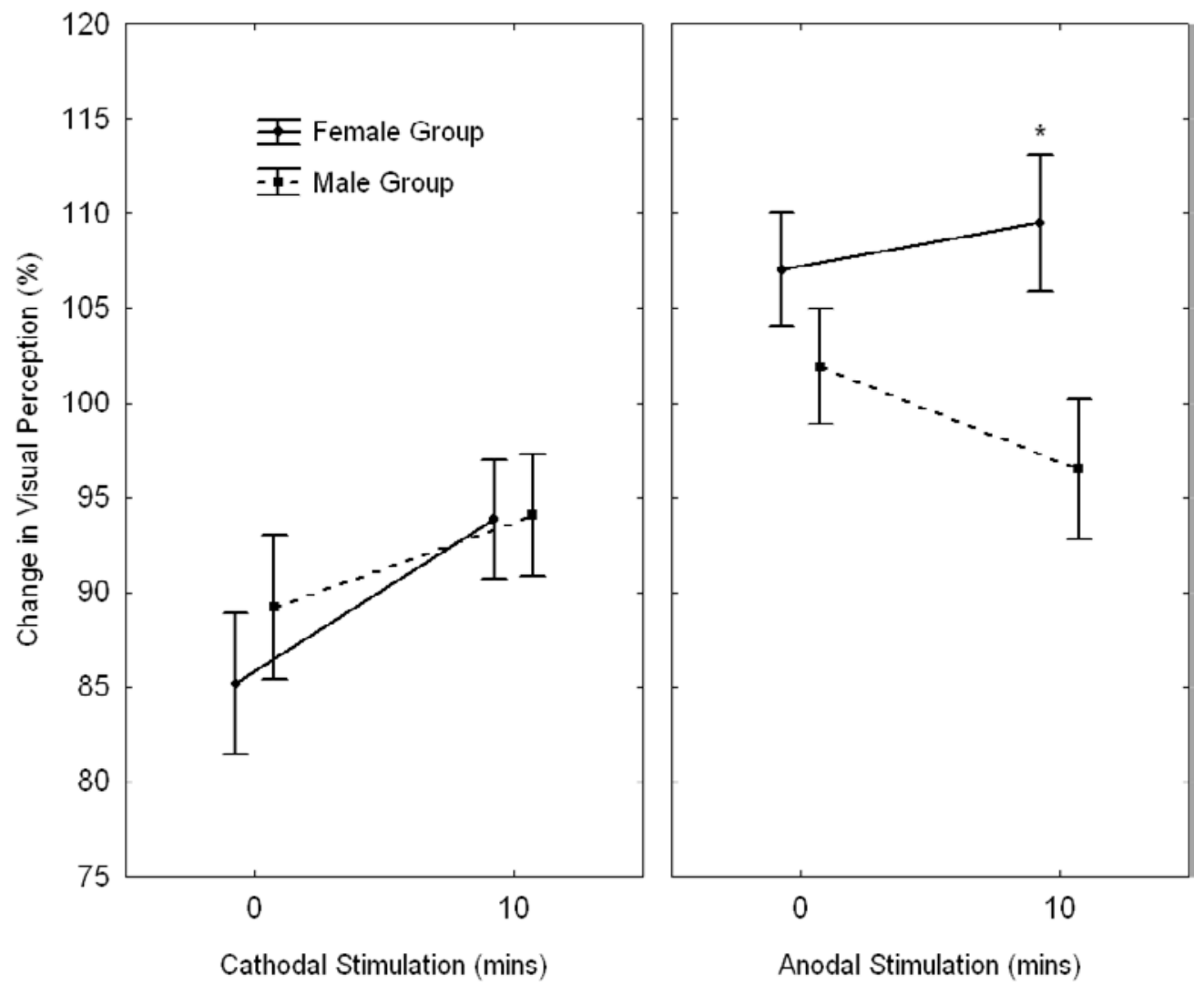

Fig. 1. Change in visual perception (\%) indicates all measurements (CS, PT, and VEPs), which are standardized and averaged. Anodal stimulation indicates an overall facilitation of all parameters in the female group. Cathodal stimulation shows no significant difference in effect between male and female subjects. The male group anodal tDCS results suggest an inhibition of all measurement parameters, increassing in effect after $10 \mathrm{~min}$ post-stimulation. Asterisks indicate results with a significant difference $(\rho \leq 0.05)$ between genders. $100 \%$ on the $\mathrm{x}$-axis is baseline; Error bars indicate SE.

The repeated measures ANOVA revealed a significant main effect of STIMULATION $(F(1,44)=28.8, p<0.0005)$, the interaction of GENDER and STIMULATION $(F(1,44)=5.1$, $p<0.05)$, and the interaction of STIMULATION and TIME $(F(1,44)=4.4, p<0.05)$ (see Table 3$)$.

The $t$-test did not reveal a significant difference between female and male subjects immediately after stimulation (cathodal stimulation: $t=-0.68, p=0.49, d f=44$; anodal stimulation $t=0.01$, $p=0.98, d f=44)$. Similarly, after $10 \mathrm{~min}$ post-stimulation for cathodal tDCS, there was no significant difference between the response of the male and female groups $(t=1,2, p=0.24, d f=$ $44)$, however, for anodal stimulation, a significant difference was observed $(t=2.54, p=0.01, d f=44)$ : female subjects were showing a higher facilitation than male subjects.

A one-way ANOVA was performed on the age variable, as this may have an influence on tDCS effects; there was no significant difference found between the two sexes, so age cannot be considered a factor in the differential response seen in tDCS-induced neuroplasticity.

\section{Discussion}

Our analysis showed that tDCS induced neuroplasticity in the human brain is partially influenced by gender-effects. No signifi-
Table 2. Means of \% change in cortical excitability with regard to the different tasks and stimulation direction ${ }^{\mathrm{a}}$

\begin{tabular}{lrr}
\hline \hline Stimulation direction & $\begin{array}{c}\text { Males } \\
\text { Mean }\end{array}$ & $\begin{array}{c}\text { Females } \\
\text { Mean }\end{array}$ \\
\hline CS & & \\
Anodal 0 & 101.7 & 100.6 \\
Cathodal 0 & 95.3 & 94.0 \\
Anodal 10 & 102.6 & 101.3 \\
Cathodal 10 & 99.7 & 100.3 \\
PTs & & \\
Anodal 0 & 109.5 & 109.2 \\
Cathodal 0 & 95.3 & 84.7 \\
Anodal 10 & 110.5 & 116.6 \\
Cathodal 10 & 96.2 & 94.0 \\
VEPs & & \\
Anodal 0 & 93.1 & 109.6 \\
Cathodal 0 & 71.8 & 104.9 \\
Anodal 10 & 85.9 & 85.0 \\
Cathodal 10 & 88.6 & \\
\hline \hline
\end{tabular}

${ }^{a}$ Means of $\%$ change in cortical excitability with regard to the different tasks and stimulation direction. Means for both measurements immediately and 10 min post-stimulation, for males and females are listed. Note that with respect to PTs an inverse calculation was used (See Materials and methods). 
Table 3. Results of repeated measures ANOVA for both anodal and cathodal stimulation directions, for 0 and $10 \mathrm{~min}$ post-stimulation $^{\text {a }}$

\begin{tabular}{lcl}
\hline \hline & F & $p$ \\
\hline Gender & 1.44 & 0.23 \\
Stimulation & 28.8 & 0.000 \\
Gender $\times$ Stimulation & 5.17 & 0.028 \\
Time & 1.36 & 0.25 \\
Gender $\times$ Time & 1.66 & 0.20 \\
Stimulation $\times$ Time & 4.61 & 0.04 \\
Gender $\times$ Stimulation $\times$ Time & 0.28 & 0.6
\end{tabular}


stimulation directions, for 0 and 10 min post-stimulation, for all parameters constituting "change in visual perception."

cant correlation between cathodal tDCS immediately after or 10 min post-stimulation was found; post-stimulation effects of anodal polarity were significantly different in the male versus female test groups. The female subjects immediately after and 10 min post-stimulation showed a facilitation of the stimulated cortical network. Males, however, showed an inhibition at the 10 min post-stimulation measurement. The tDCS induced inhibition or facilitation is a transient stage of induced short-term neuroplasticity closely related to changes in the state of the neuronal membrane. Ardolino et al. (2005) describe the potential mechanisms of tDCS in their paper examining the non-synaptic after-effects of DC stimulation on peripheral motor axons and neurons in the central nervous system. The migration of transmembrane proteins or changes in ion concentration across the membrane, in response to a continuous electrical field, may be responsible for the short-term neuroplastic effects; suggesting a possible mechanism of tDCS action on the human cortex. Further evidence can be derived from pharmacological studies also using tDCS. Nitsche et al. $(2003 b)$ demonstrated that the application of sodium and calcium ion channel blockers are able to attenuate both the immediate and longer term after-effects of anodal stimulation, while NMDA receptor blockers (glutamate) inhibit the development of long-term effects of tDCS, regardless of stimulation polarity (Liebetanz et al., 2002). These findings indicate that the short-term neuroplastic effects arise from alterations in neuronal membrane function and longer-term effects are mediated at the level of the synapse.

A similar study (Kuo et al., 2006) recently showed a reverse effect with respect to gender-bias, on MEPs measured from the primary motor cortex in males and females. The primary motor cortex relays information from the central nervous system to the periphery. However, the primary visual cortex is activated by input mechanisms, taking cues from the external environment, and relaying them to other cortical areas in order to be processed. Their cortical projections and efferents originate in and from differing neuroanatomical structures, and as a result, may possess contrasting neurotransmitter receptors. The origins of each of the parameters of visual perception are also differing and complex; VEPs are a measure of the cortical response to thalamic inputs, PTs indicate cortical background (V1-V2-V3) activity, while CSs mainly arise from the simultaneous activity of intracortical inhibitory processes at the spatial level of cortical receptive fields. By cross-analyzing each parameter, we were able to gain an overall effect of tDCS on visual perception, and use this to compare responses between the sexes. Therefore gender differences are related to a comparison between all parameters of visual perception between male and female groups.

This study suggests that there may exist, alternating pathways of neuroplasticity between the sexes in the visual cortex. The influences of gonadal sex hormones, neurotrophins, and the presence of neurotransmitter receptors on neural membranes may all be influential in the modulation of neuroplasticity, and may be expressed differently within each gender.

Inghilleri et al. (2004), using a repetitive transcranial magnetic stimulation (rTMS) paradigm, found that estrogens are able to influence cortical excitability; low estrogen levels have a damping effect on sodium channels which reduces the recruitment of excitatory interneurons, thus reducing cortical excitability, whereas at higher levels estrogens increase cortical excitability. In addition to this, Smith et al. $(2002 a, 2002 b)$ reported that progesterone has a reverse effect, and increases inhibition in neuronal networks by modulating GABAergic transmission; having earlier conducted one of the earliest studies to demonstrate menstrual cycle effects on cortical excitability (Smith et al., 1999). Studies in vitro have mirrored findings from in vivo investigations; Smith et al. (2002a, $2002 b$ ) examined fluctuations in gonadal hormone levels in organotypic hippocampal slices, in addition to using a kindling paradigm, and found that slices from intact males showed a greater level of baseline excitability than those of the female rats. They concluded that higher levels of testosterone may have an excitatory effect on hippocampal responses. In a recent review, Cooke and Woolley (2005) discuss the effect of gonadal steroids, androgen, and estrogen, on the morphology of dendrites forming synaptic connections. The pyramidal cells of the CA1 rat hippocampus undergo morphological changes in phase with the estrous cycle, suggesting that neuroplasticity on a synaptic level is influenced by fluctuations in steroid hormones. Earlier studies by Rudick and Woolley (2001) showed evidence of NMDA and $\mathrm{GABA}_{\mathrm{A}}$ estrogen mediated input to CA1 pyramidal cells, restoring the excitatory and inhibitory balance and increasing pyramidal cell responses to synaptic input.

If gonadal hormones are, on a molecular level, modulating synaptic connections, then the excitatory and inhibitory intracortical circuitry are also being modified. A study by Hausmann et al. (2006) investigated the effects of estradiol and progesterone on transcallosal inhibition. They concluded that these steroid hormones modulated the iSPs in accordance with each phase of the menstrual cycle, and that they were able to alter functional cerebral organization via activation of glutamatergic or GABAergic neurons. Maguire et al. (2005) demonstrated cycling expression of $\mathrm{GABA}_{\mathrm{A}}$ receptor subunits in hippocampal neurons mediating tonic inhibition during the ovarian cycle, altering seizure susceptibility and anxiety in mice.

Another intriguing aspect of gonadal hormones' involvement in neuromodulation is the rare incidences of migraine in males, in comparison to the statistical prevalence in females. A case review by Brandes (2006) discussed how the influence of estrogen on migraine is three-fold greater amongst women than men, and how the prevalence changes with reproductive status. The author also reports that menstrual migraine is pharmacologically resistant and without aura, whereas male migraineurs often experience aura even without headache.

In addition to differences in hormonal expression between the sexes, there appears to be evidence of differences in cortical structure. Recent studies have shown that in the rat visual cortex 
there exist dimorphic differences governed by gender. Nunez et al. (2002) showed that ovarian steroids expressed after postnatal day 20 reduce the number of neurons in the binocular region of the primary visual cortex of the female rat. Pakkenberg and Gundersen (1997) showed in an earlier study performed on human cadavers that on average females possess $16 \%$ fewer neocortical neurons than males, even though neuronal cortical density remains the same.

Understanding how the induction of short-term neuroplasticity in the mammalian cortex is achieved belies the understanding of neuronal excitability differences between males and females. The variation in background hormone levels and in cortical structure between males and females may account for the different observed responses to tDCS application. These findings, along with many other studies describing the influences of sex on plasticity, suggest that gender differences should be accounted for when evaluating changes in neuroplasticity, both short and long-term. However, more focused studies are required to elucidate the explicit mechanisms of gender-effects on neuroplasticity.

\section{Conclusion}

The current findings suggest that there exists gender-bias in the primary visual cortex which manifests itself in the altered excitability levels between the sexes resulting in a non-synaptic mechanism of neuromodulation in membrane function.

\section{References}

Antal, A., Kincses, T.Z., Nitsche, M.A., Bartfai, O. \& Paulus, W. (2004). Excitability changes induced in the human primary visual cortex by transcranial direct current stimulation: Direct electrophysiological evidence. Investigative Ophthalmology \& Visual Science 45, $702-707$.

Antal, A., Kincses, T.Z., Nitsche, M.A. \& Paulus, W. (2003). Manipulation of phosphene thresholds by transcranial direct current stimulation in man. Experimental Brain Research 150, 375-378.

Antal, A., Nitsche, M.A. \& Paulus, W. (2001). External modulation of visual perception in humans. NeuroReport 12, 3553-3555.

Ardolino, G., Bossi, B., Barberi, S. \& Priori, A. (2005). Non-synaptic mechanisms underlie the after-effects of cathodal transcutaneous direct current stimulation of the human brain. Journal of Physiology 568 , 653-663.

Bindmann, L.J., LiPPOLd, O.C.J. \& REDFEARN, J.W.T. (1964). The action of brief polarizing currents on the cerebral cortex of the rat (I) during current flow and (2) in the production of long-lasting after-effects. Journal of Physiology 172, 369-382.

Boroojerdi, B., Meister, I., Foltys, H., Sparing, R., Cohen, L. \& TöPPER, R. (2002). Visual and motor cortex excitability: A transcranial magnetic stimulation study. Clinical Neurophysiology 113, 1501-1504.

BRANDES, J.L. (2006). The influence of estrogen on migraine. JAMA 295, $1824-1830$.

CAHILl, L. (2006). Why sex matters for neuroscience. Nature Reviews Neuroscience 7, 477-484.

Cooke, B.M. \& Woolley, C.S. (2005). Gonadal hormone modulation of dendrites in the mammalian CNS. Neurobiology 64, 34-46.

Hausmann, M., Tegenthoff, M., Sänger, J., Janssen, F., Güntürkün, O. \& Schwenkreis, P. (2006). Transcallosal inhibition across the menstrual cycle: A TMS study. Clinical Neurophysiology 117, 26-32.
Inghilleri, M., Conte, A., Curra, A., Frasca, V., Lorenzano, C. \& Berardelli, A. (2004). Ovarian hormones and cortical excitability. An rTMS study in humans. Clinical Neurophysiology 115, 1063-1068.

JuRASKA, J.M. (1998). Neural plasticity and the development of sex differences. Annual Review of Sex Research 9, 20-38.

Keller, A. (1993). Intrinsic synaptic organization of the motor cortex. Cerebral Cortex 3, 430-441.

Kimura, D. (2002). Sex hormones influence human cognitive pattern. Neuroendocrinology Letters 23, 67-77.

Kuo, M., Paulus, W. \& Nitsche, M.A. (2006). Sex differences in cortical neuroplasticity in humans. NeuroReport 17, 1703-1707.

Lang, N., Siebner, H.R., Ward, N.S., Lee, L., Nitsche, M.A., Paulus, W., Rothwell, J.C., Lemon, R. \& Frackowiak, R.S. (2005). How does transcranial DC stimulation of the primary motor cortex alter regional neuronal activity in the human brain? European Journal of Neuroscience 22, 495-504.

Liebetanz, D., Nitsche, M.A., Tergau, F. \& Paulus, W. (2002). Pharmacological approach to the mechanisms of transcranial DC-stimulationinduced after-effects of human motor cortex excitability. Brain 125, $2238-2247$.

LUND, J.S. (2002). Specificity and non-specificity of synaptic connections within mammalian visual cortex. Journal of Neurocytology 31, 203-209.

Maguire, J.L., Stell, B.M, Rafizadeh, M. \& Mody, I. (2005). Ovarian cycle-linked changes in $\mathrm{GABA}_{\mathrm{A}}$ receptors mediating tonic inhibition alter seizure susceptibility and anxiety. Nature Neuroscience 8, 797-804.

Nitsche, M.A. \& Paulus, W. (2000). Excitability changes induced in the human motor cortex by weak transcranial direct current stimulation. Journal of Physiology 52, 633-639.

Nitsche, M.A. \& Paulus, W. (2001). Sustained excitability elevations induced by transcranial DC motor cortex stimulation in humans. $\mathrm{Neu}$ rology 57, 1899-1901.

Nitsche, M.A., Fricke, K., Henscke, U., Schlitterlau, A., Liebetanz, D., Lang, N., Henning, S., Tergau, F. \& Paulus, W. (2003a) Pharmacological modulation of cortical excitability shifts induced by transcranial direct current stimulation in humans. Journal of Physiology 553, 293-301.

Nitsche, M.A., Lampe, C., Antal, A., Liebetanz, D., Lang, N., TerGaU, F. \& Paulus, W. (2006). Dopaminergic modulation of longlasting direct current-induced cortical excitability changes in the human motor cortex. European Journal of Neuroscience 23, 1651-1657.

Nitsche, M.A., Nitsche, M.S., Klein, C.C., Tergau, F., Rothwell, J.C. $\&$ PAulus, W. (2003b). Level of action of cathodal DC polarisation induced inhibition of the human motor cortex. Clinical Neurophysiology 114, 600-604.

Nunez, J.L., SoDhI, J. \& JuRAsKa, J.M. (2002). Ovarian hormones after postnatal day 20 reduce neuron number in the rat primary visual cortex. Journal of Neurobiology 52, 312-321.

Pakkenberg, B. \& Gundersen, H.J.G. (1997). Neocortical neuron number in humans: Effect of sex and age. The Journal of Comparative Neurology 384, 312-320.

Rudick, C.N. \& Woolley, C.S. (2001). Estrogen regulates functional inhibition of hippocampal CA1 pyramidal cells in the adult female rat. Journal of Neuroscience 21, 6532-6543.

Sherman, S.M. \& Spear, P.D. (1982). Organization of visual pathways in normal and visually deprived cats. Physiology Review 62, 738-855.

Smith, M.D., Jones, L.S. \& Wilson, M.A. (2002a). Sex differences in hippocampal slice excitability: Role of testosterone. Neuroscience $\mathbf{1 0 9}$, 517-530.

Smith, M.J., Adams, L.F., Schmidt, P.J., Rubinow, D.R. \& Wassermann, E.M. (2002 b). Effects of ovarian hormones on human cortical excitability. Annals of Neurology 51, 599-603.

Smith, M.J., Keel, J.C., Greenberg, B.D., Adams, L.F., Schmidt, P.J., Rubinow, D.A. \& Wasserman, E.M. (1999). Menstrual cycle effects on cortical excitability. Neurology 53, 2069-2072. 